\title{
Indicadores estáticos normalizados para estabilidad de tensión basados en reservas de potencia reactiva y en márgenes de cargabilidad
}

\author{
Normalized static indexes for voltage stability based on reactive power reserves \\ and loadability margins
}

\author{
Jorge W. González ${ }^{1} \quad$ Hugo A. Cardona ${ }^{1} \quad$ Idi A. Isaac $^{1} \quad$ Gabriel J. López $^{1}$
}

Recibido 3 de octubre de 2013, aceptado 30 de julio de 2014

Received: October 3, $2013 \quad$ Accepted: July 30, 2014

\begin{abstract}
RESUMEN
En este artículo se proponen indicadores normalizados, o en por unidad, para análisis de estabilidad de tensión en un sistema eléctrico de potencia. Los indicadores son simples y se conciben en función de la potencia reactiva de reserva y del margen de cargabilidad del sistema. La metodología presentada con los indicadores propuestos hace posible conjugar la evolución del margen de cargabilidad y las reservas de potencia reactiva. Esto es particularmente útil o beneficioso para analistas y operadores al permitir que se interprete de manera adecuada si la presencia de potencia reactiva de reserva es una garantía de la estabilidad de tensión o no en determinado punto del sistema de potencia. Los indicadores se aplicaron con éxito, exponiendo tendencias similares para una red IEEE pequeña ampliamente conocida y para una red de gran cantidad de barras y generadores. Por otro lado, a través de una arquitectura básica propuesta para la integración de tecnologías de información, se indicó la conveniencia del uso de indicadores para aplicaciones modernas de sincrofasores y conciencia situacional, en la que los operadores y analistas podrán contar con información precisa de lo que ocurre en el sistema y concebir probables soluciones con éxito.
\end{abstract}

Palabras clave: Estabilidad de tensión, indicadores estabilidad, reserva de potencia reactiva, margen de carga, sincrofasores, conciencia situacional.

\section{ABSTRACT}

In this paper, normalized or per unit indices for voltage stability analysis in power systems are proposed. The indices are simple and designed based on reactive power reserves and system loadability margins. The methodology presented with the proposed indices makes it possible to combine the loadability margin evolution and reactive power reserves. The method is particularly useful or beneficial for analysts and operators, since it helps to properly interpret if the presence of reactive power reserve is a guarantee of the stability of voltage in a given location of the power system. The indices were successfully applied, exposing similar trends for an IEEE small network, which is widely known, and for a network having a large number of busbars and generators. On the other hand, through a basic architecture proposed for the integration of information technologies, the convenience of indices usage for modern applications of Synchrophasors and Situational Awareness has been pointed out, in which operators and analysts may count on accurate information about the evolution of the power system and to successfully conceive solutions.

Keywords: Voltage stability, stability indexes, reactive power reserve, loadability margin, sinchrophasors, situational awareness.

1 Universidad Pontificia Bolivariana. Grupo de Investigación en Transmisión y Distribución (Grupo TyD). Circular 1 N 70-01. Medellín, Colombia.

E-mail: jorgew.gonzalez@upb.edu.co; hugo.cardona@upb.edu.co; idi.isaac@upb.edu.co; gabriel.lopez@upb.edu.co 


\section{INTRODUCCIÓN}

En los últimos años se han presentado en el mundo múltiples eventos relacionados con la estabilidad de tensión. Dichos eventos han ocasionado incidentes en cascada desconectando altas cantidades de usuarios [1]. Debido a lo anterior, la estabilidad de tensión se ha convertido en un tema clave de estudio [2]. Muchas han sido las metodologías y en particular los indicadores propuestos sobre este tipo de estabilidad. Unas primeras sensibilidades basadas en variaciones de tensiones, potencias y carga fueron presentadas en [3-4]. Para los análisis ha sido clásico el uso de métodos estáticos en forma de curvas P-V y Q-V. Incluso, a partir de dichos métodos se ha logrado construir indicadores de estabilidad de tensión [3]. Dichos indicadores también sirven para un análisis en tiempo real que juzgue la evolución del sistema de potencia.

Para estudios de estabilidad de tensión es fundamental tener en cuenta los límites de capacidad de potencia reactiva de los generadores sincrónicos $[2,5]$. La cantidad de reservas de potencia reactiva impacta directamente los márgenes de estabilidad de tensión, por lo que se debe ejercer un manejo adecuado de estas reservas [6].

Según [7], los avances en la estimación de márgenes de estabilidad de tensión monitoreando las reservas de potencia reactiva son aún insuficientes. Este inconveniente aplica tanto para los análisis de planeamiento operativo como para tiempo real.

Sobre la literatura de alto nivel relacionada con el tema de estabilidad de tensión y márgenes de reserva de potencia reactiva, en [7] se aborda el problema a través de las regresiones lineales considerando una exhaustiva cantidad de casos. De esta forma se obtienen relaciones sobre el margen de carga y las reservas de potencia reactiva.

En [8], el objetivo es determinar la ubicación y cantidad de acciones de control que permitan restablecer los márgenes de potencia reactiva críticos en el sistema eléctrico de potencia. En dicho trabajo se abordó un problema de optimización convexo cuadrático.

En [9] se proponen las mejoras sobre el margen de estabilidad de tensión con reformas en el despacho económico, de forma indirecta empleando factores de participación a partir de valores propios críticos de la matriz Jacobiana en el máximo punto de carga. El método no utiliza indicadores para la medida de la distancia al punto de colapso de tensión. La aplicación no fue concebida para desempeños en tiempo real.

Con base en la evolución de las reservas de potencia reactiva de los generadores y en los márgenes de carga, en este artículo se proponen indicadores para el análisis de estabilidad de tensión, bien sea para aplicaciones durante el planeamiento operativo o para utilización en tiempo real. La novedad de los indicadores propuestos, es que estos son estáticos en un marco referencial normalizado o en por unidad, lo que permitirá la comparación paralela entre márgenes de carga y reservas de potencia reactiva en un sistema eléctrico de potencia. Esta solución no se encuentra en la literatura de estabilidad de tensión. De otro lado, se introducirá cómo podrían integrarse los indicadores propuestos en visualizaciones desarrolladas bajo conceptos de Conciencia Situacional (SA del inglés Situational Awareness). Es de aclarar que el concepto de SA está siendo ampliamente concebido en la actualidad para su uso generalizado en aplicaciones de potencia eléctrica, dada la masificación de unidades de medición fasorial o sincrofasores (PMU del inglés Phasor Measurement Units) [10-15].

Con los indicadores y una metodología propuesta, que explota las ventajas de las PMU, se podrá encontrar cómo es posible ayudar en la operación de los sistemas eléctricos garantizando la supervisión de la estabilidad de tensión a la par con las reservas de potencia reactiva. El método propuesto es sencillo y no requiere mapear grandes cantidades de casos como se presenta en [7-8], sino más bien realizar la predicción del margen de carga del sistema y de las reservas de potencia reactiva, todo en un marco de referencia común en por unidad.

Una comprobación o validación del método propuesto será que la característica de reserva de potencia posee una región lineal seguida de una no lineal en la zona de mayor estrés, como se expuso en $[7,16]$.

El método con los indicadores propuestos fue implementado y comprobado en un reconocido programa de simulación digital para la red IEEE de 
nueve barras, que posee tres generadores. También se comprobó en el sistema eléctrico colombiano, el que es un caso de grandes dimensiones, ya que contiene más de 2200 barras y más de 350 unidades de generación.

El resto del artículo está organizado de la siguiente forma: la segunda sección presenta los fundamentos de reservas de potencia reactiva en generadores sincrónicos, la tercera y cuarta sección exponen la obtención de los indicadores propuestos, la quinta contiene los resultados de simulación, la sexta sección es una introducción sobre potencialidades de aplicación con sincrofasores en el marco de la Conciencia Situacional. Finalmente, se presentan las conclusiones del trabajo en la séptima sección.

\section{RESERVAS DE POTENCIA REACTIVA DE GENERADORES}

De manera primordial, la característica de potencia reactiva de los generadores síncronos está limitada por la capacidad de la armadura y del campo [2-3]. Para una potencia activa dada, la generación de potencia reactiva posee límites de armadura y de campo, como lo exponen las ecuaciones (1) y (2) de manera respectiva.

$$
\begin{gathered}
Q_{\text {gmáx_a }}=\sqrt{\left(V_{g}^{2} I_{a}^{2}-P_{g}^{2}\right)} \\
Q_{\text {gmáx_f }}=-\frac{V_{g}^{2}}{X_{S}}+\sqrt{\left(\frac{V_{g}^{2} I_{f}^{2}}{X_{S}^{2}}-P_{g}^{2}\right)}
\end{gathered}
$$

El menor de los valores establece la máxima potencia reactiva de salida. Sin embargo, es usual que los operadores dispongan de un límite constante de potencia reactiva, tanto para consideraciones de operación como de planeamiento operativo. Esto frecuentemente está en consonancia con ajustes de protecciones de sobrecorriente en las protecciones de generación [17]. Por otro lado, también es típico que los estudios de flujo de cargas se realicen considerando un valor constante límite de potencia reactiva, a menos que se disponga de la característica precisa del generador. En este trabajo se utilizará un límite constante para mostrar la tendencia de comportamiento de los indicadores propuestos.

La Figura 1 presenta el primer cuadrante de la característica de capacidad típica de un generador sincrónico [2]. En la Figura 1 es posible definir la Reserva de Potencia Reactiva, RPR, como la diferencia entre el límite de reactiva y el valor de reactiva actual, como lo presenta la ecuación (3).

$$
Q_{R P R}=Q_{C}-Q_{a c t u a l}
$$

A continuación se lista la notación de términos de las ecuaciones (1) a (3).

$Q_{\text {gmáx_a }} \quad$ Máxima potencia reactiva de salida de un generador-límite de armadura

$Q_{\text {gmáx_f }} \quad$ Máxima potencia reactiva de salida de un generador-límite del campo

$Q_{R P R} \quad$ Potencia reactiva de reserva

$Q_{C} \quad$ Límite constante de potencia reactiva

$Q_{\text {actual }}$ Valor actual de potencia reactiva durante la operación

$V_{g} \quad$ Tensión terminal del generador

$I_{a} \quad$ Máxima corriente de armadura de un generador

$I_{f} \quad$ Máxima corriente de campo de un generador

$P_{g} \quad$ Potencia activa de salida de un generador

$X_{S} \quad$ Reactancia sincrónica de un generador

En este trabajo las cargas se modelarán como del tipo de potencia constante. El margen de cargabilidad se define como la cantidad de potencia activa que se puede entregar a una carga antes de alcanzar el punto de colapso en la tensión. Este margen se mide a partir de una potencia definida como inicial, ver Figura 2. El punto de colapso, o punto teórico de máxima transferencia de potencia, se conoce como punto de máxima cargabilidad. Las curvas de la Figura 2 corresponden a las curvas $\mathrm{P}-\mathrm{V}$ de estabilidad de tensión y son comúnmente empleadas para este tipo de estudios, principalmente desde un punto de vista estático. Otro indicador que podría emplearse es el ATC (ATC del inglés Available Transfer Capability), que especifica límites extremos máximos para intercambio de potencia con explotación comercial, conservando en este caso la tensión en rangos admisibles por los entes reguladores.

En el actual trabajo se empleará el análisis del margen de cargabilidad con el objetivo de realizar un trazado completo de los indicadores que se presentarán. El trazado irá desde el caso base hasta el punto del 


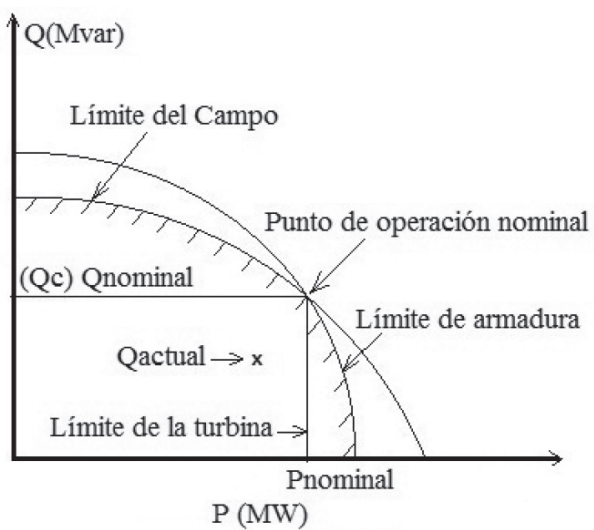

Figura 1. Curva de capacidad típica de un generador sincrónico. Definiciones de límites de potencia reactiva.

colapso o punto teórico. Esto permitirá realizar un seguimiento completo a la disminución de las reservas de potencia reactiva en el sistema, hasta el punto teórico de la máxima potencia transferida.

Las curvas P-V se construyen variando la carga en los nodos de interés. Cada carga i es modificada de acuerdo con un parámetro de carga [4].

En la Figura 2 también se ha incluido una curva que presenta cómo el margen de cargabilidad se disminuye debido a una contingencia en el sistema de potencia. El comparativo se realizó con base en una misma potencia inicial o actual de la carga de interés $i$.

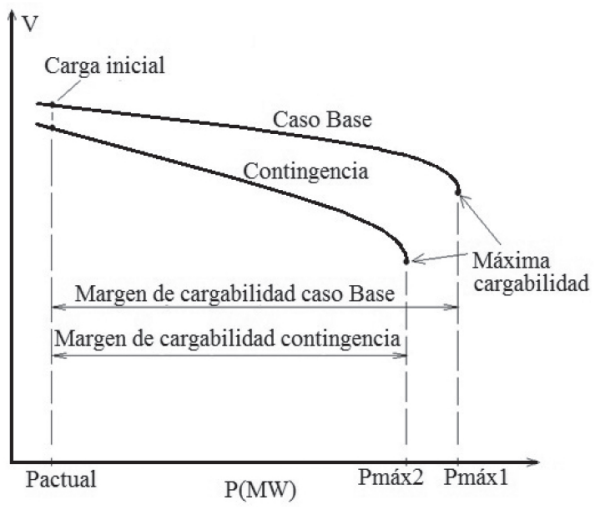

Figura 2. Curvas P-V con definición del Margen de cargabilidad para una carga $i$.

Según la referencia [18], en los estudios de estabilidad de tensión de largo plazo, un colapso de tensión puede ocurrir a partir del incremento constante de la carga y seguido de un agotamiento en las reservas de potencia reactiva. Una consecuencia sería la pérdida del control de la tensión o la salida de equipos por acción de sistemas de protección.

En $[7,16]$ se reportó sobre la existencia de una relación casi lineal entre el margen de cargabilidad y las reservas de potencia reactiva en los generadores. La relación anterior confirma que dichas reservas de potencia podrían ser empleadas como indicadores de estabilidad de tensión. Por otro lado, una importante cantidad de metodologías que introducen los requerimientos de estabilidad de tensión en el flujo de cargas óptimo proponen una optimización multiobjetiva basada en el margen de cargabilidad, en los valores propios mínimos, en valores singulares mínimos, entre otros [9, 19]. El inconveniente es que en la cercanía al punto de colapso, los indicadores mencionados poseen un comportamiento no lineal. Este problema de no linealidad conlleva a dificultades en la convergencia de estos métodos y a su éxito en sistemas de potencia grandes con altos niveles de carga [20].

Se proponen a continuación unos indicadores normalizados para el margen de carga y de reserva de potencia reactiva. Estos indicadores son sencillos en su definición y fáciles de obtener. Se resalta que para un sistema de potencia que incluya sincrofasores (PMU), para la estimación de estados, los resultados de los indicadores serían más precisos, permitiendo una mejor toma de decisiones para los analistas y operadores. Según la norma IEEE C37.118, partes 1 y 2 [21-22], es posible obtener magnitudes de tensión, ángulos, potencia reactiva, etc., en el punto de instalación mediante la medición con sincrofasores.

Por otro lado, si se cuenta con una estimación de estados concebida con sincrofasores, el éxito de los métodos propuestos para estabilidad de tensión será de mayor probabilidad [23].

\section{INDICADOR DE RESERVA DE POTENCIA REACTIVA}

Este indicador será normalizado, lo que permitirá una comparación en un mismo sistema coordenado con el segundo indicador. El indicador se denominará $Q_{\text {retp }}^{j}$ y aplica para cada barra $i$ durante el estado de carga $j$. Ver ecuación (4). Se debe tener en cuenta que en el sistema habrá generadores despachados y 
no despachados. Estos últimos no intervendrán en la reserva de potencia reactiva "rodante" durante los análisis de la situación de estabilidad de tensión. El indicador permitirá el monitoreo de las reservas de potencia reactiva para cada situación de carga en una barra de interés. El subíndice $p$ significa valor normalizado o en por unidad. El subíndice $c$ corresponde al término carga. El sistema redcurva de la Figura 3 permite aclarar la definición de parámetros y condiciones para los indicadores que se proponen.

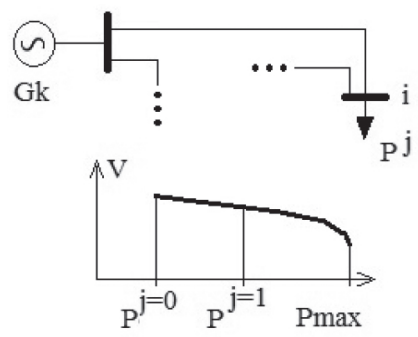

Figura 3. Definiciones de red y curva P-V para los indicadores propuestos en el estado de carga $j$.

$$
Q_{r e t p}{ }_{i}^{j}=\frac{Q_{r t}^{j} / P_{c i}^{j}}{Q_{r t}^{0} / P_{c i}^{0}}
$$

En esta expresión,

$$
Q_{r t}^{j}=\sum_{k \in \alpha_{L}}^{n} Q_{R P R_{k}}
$$

$k=1,2,3, \ldots, n$ generadores

$\alpha_{L}=\{$ Generadores despachados $\}$

$$
\begin{gathered}
Q_{R P R_{k}}=Q_{C_{k}}-Q_{\text {actual }_{k}} \quad \forall x \in \alpha_{L} \\
Q_{r t}^{0}=\underbrace{\max }_{k \in \alpha_{L}}\left\{Q_{R P R_{k}}\right\}
\end{gathered}
$$

$P_{c i}^{j}$ : Es la potencia actual, en el estado de carga $j$, medida en la barra de interés $i$.

$P_{c i}^{0}:$ Corresponde a la potencia activa nominal de la carga en la barra de estudio $i$. Coincide normalmente con la potencia base de la carga.

$Q_{r t}^{j}$ : Es la reserva total de potencia reactiva en el estado de carga $j$.
$Q_{r t}^{0}:$ Reserva de potencia reactiva respecto del caso base o de referencia. Coincide con la máxima reserva de potencia reactiva de estudio inicial. Esta potencia normalizadora, al ser tomada en el caso inicial sin contingencias, poseerá el mayor valor de reserva de potencia reactiva con relación a los otros estudios.

\section{INDICADOR DE MARGEN DE CARGABILIDAD}

Este indicador también será normalizado y monitoreado con $Q_{r e t p}^{j}$ en un mismo diagrama coordenado para cada condición de carga.

El indicador permitirá monitorear el margen de cargabilidad para cada estado de carga $j$ en la barra de interés $i$. Este indicador se denominará $M_{\text {cap } i}{ }_{j}^{j} \mathrm{y}$ aplica para cada barra $i$ estudiada según la ecuación (7). Para obtener el valor de este indicador se requiere conocer la potencia máxima para la barra $i$ en el punto de colapso de tensión, $P_{\text {máxi }}$. Esto se calcula durante etapas de planeamiento operativo para los escenarios de interés, que incluyen el caso base, contingencias probables y respuestas ante soluciones a las contingencias.

$$
M_{c a p i}^{j}=\frac{P_{m a ́ x}-P_{c i}^{j}}{P_{m a ́ x}-P_{c i}^{0}}
$$

En la sección siguiente se realizarán las simulaciones que expliquen la aplicación y beneficios de los indicadores propuestos.

\section{SIMULACIONES Y ANÁLISIS DE RESULTADOS}

Los indicadores propuestos se han simulado en la red estándar IEEE de 9 barras [24] y en una red grande. Para este último caso se utilizó la red colombiana. Las simulaciones fueron ejecutadas en el programa DIgSILENT PF [25]. Los resultados de las simulaciones fueron exportados a hojas de cálculo y posteriormente graficados.

Para construir las curvas $\mathrm{P}-\mathrm{V}$ en barras seleccionadas, el DIgSILENT incrementa las cargas manteniendo constante el factor de potencia. Las cargas se incrementan automáticamente con flujos de carga 
hasta que no se tenga convergencia. La eficiencia del algoritmo es alta debido a que el tamaño de paso de los incrementos de potencia es variable. Para realizar las curvas $\mathrm{P}-\mathrm{V}$ se emplearon las siguientes características en el programa: Tamaño mínimo y máximo de los pasos: 0,001 (1 milésima) y 1 unidad respectivamente.

\section{Sistema IEEE 9 Barras [24]}

En la Figura 4 se presenta el diagrama de la red IEEE de 9 barras. Esta red posee tres generadores. Las cargas totales son de 315 MW y 115 Mvar. Según las características de los generadores, el margen de reserva de potencia reactiva total de los tres generadores para el caso base es de 356,5 Mvar.

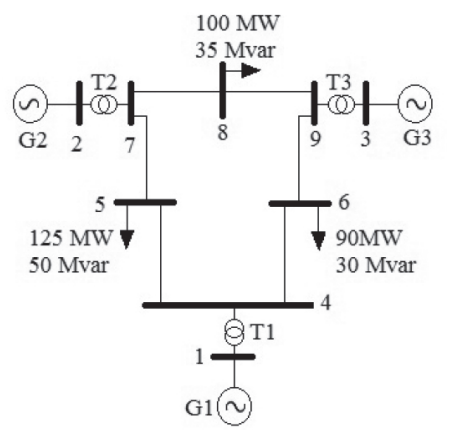

Figura 4. Sistema IEEE de 9 barras [24].

Para ilustrar la metodología que se debe seguir para la obtención y análisis de los indicadores, se eligió la barra 6 para ser monitoreada desde el punto de vista de estabilidad de tensión. La carga en esta barra será incrementada hasta el punto del colapso.

En la Figura 5 se pueden apreciar las curvas P-V para cuatro situaciones: el caso base, la contingencia de salida de la línea entre las barras 9 y $6(\mathrm{~N}-1)$, la contingencia de salida del grupo generadortransformador G2-T2 (N-2), y finalmente para el sistema de doble contingencia $(\mathrm{N}-2)$ con una compensación capacitiva de 25 Mvar que se conecta en la barra 6 .

Como era de esperarse, el valor de potencia máxima para colapso de tensión se disminuye. El peor caso se obtiene para la contingencia $\mathrm{N}-2$ con un valor de 210,8 MW. La inclusión del banco de compensación capacitiva permite una potencia máxima de 222,3 MW.

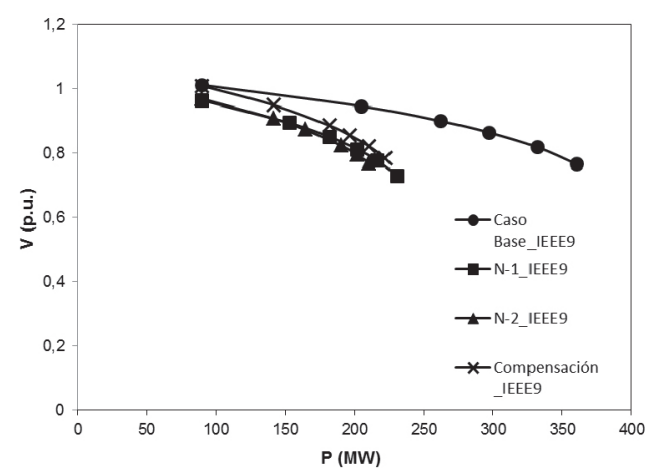

Figura 5. Curvas P-V sistema IEEE 9 barras para los casos de estudio. Barra 6.

En la Figura 6 se han dibujado las curvas de potencia reactiva de reserva total para los casos de estudio en los estados de carga que corresponden a las curvas P-V. Puede apreciarse cómo las reservas se disminuyen a medida que el sistema es estresado por medio del incremento de la demanda en la barra 6. De igual forma que para las curvas $\mathrm{P}-\mathrm{V}$, el mejor caso corresponde al base, esto por tener mejores reservas; el peor caso es para la contingencia doble. La compensación capacitiva mejora la condición ocasionada por la doble contingencia.

Nótese que las reservas de potencia reactiva no son iguales en todos los casos, es más, no son nulas. En realidad, para un nodo de carga existe un conjunto de generadores que afectan directamente su margen de estabilidad de tensión. Por lo que es de esperarse que se llegue al punto de colapso de tensión a pesar de disponerse aún de reserva de potencia reactiva en todo el sistema. Esto se encuentra acorde con

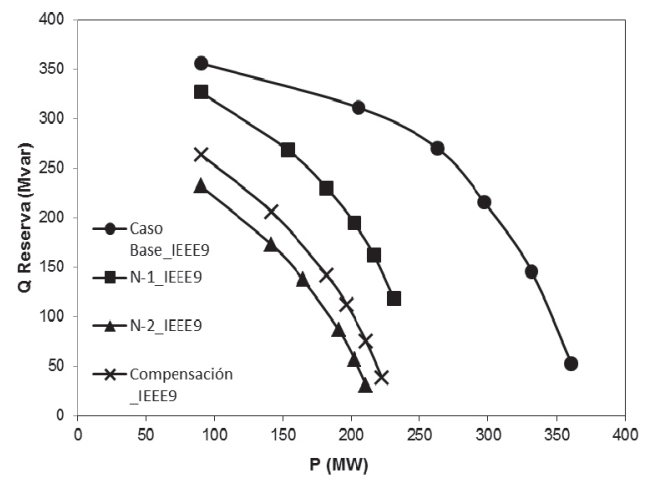

Figura 6. Potencias reactivas de reserva sistema IEEE 9 barras para los casos de estudio. Estrés en Barra 6. 
lo definido en la referencia [26], en la cual se explica que existe un número crítico reducido de generadores que al ser agotados ocasionarán el colapso de tensión. Dicha situación obliga a un estudio detallado de los generadores que conforman la reserva de potencia reactiva y su relación con los nodos de carga del sistema. Los estudios de este trabajo se han realizado considerando los límites de potencia reactiva de los generadores.

La comparación de los indicadores propuestos se puede observar en la Figura 7 para el caso base y en la Figura 8 para la contingencia de una línea mencionada previamente. Puede notarse que el indicador del margen de cargabilidad expone un comportamiento lineal, mientras que el de potencia reactiva de reserva posee una región líneal y otra con característica cuadrática. Esta relación es ventajosa para mostrar que por su definición, ambos indicadores

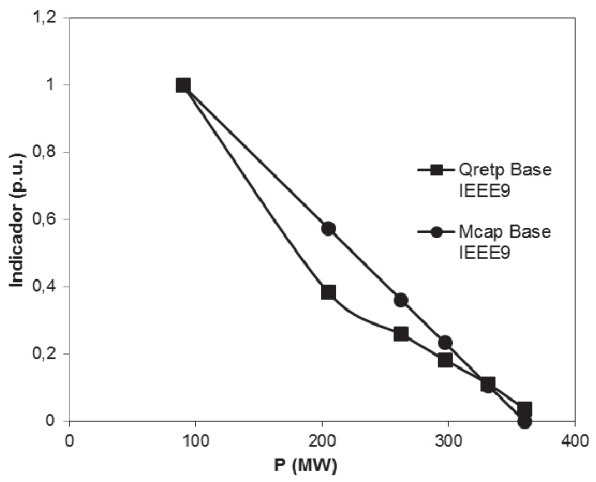

Figura 7. Comparación de indicadores para el caso base. Sistema IEEE 9 barras. Estrés en Barra 6.

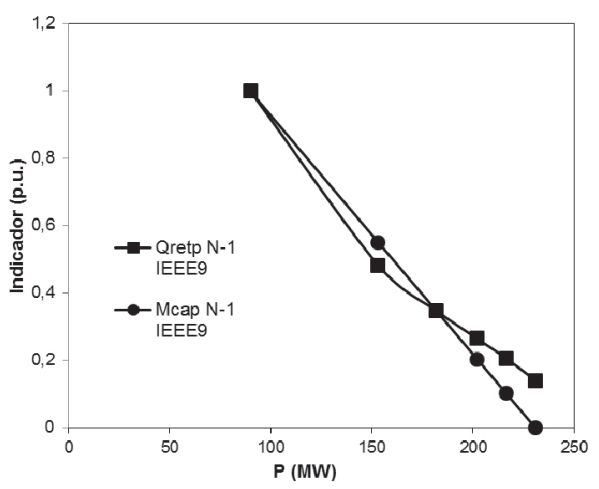

Figura 8. Comparación de indicadores para una contingencia. Sistema IEEE 9 barras. Estrés en Barra 6. son iniciados en 1 p.u., mientras que el indicador del margen de cargabilidad se hace cero cuando la potencia actual alcanza el límite máximo (margen de cargabilidad nulo).

Esta situación es útil para los analistas y operadores, ya que la identificación de la evolución de las reservas de potencia reactiva se ejecuta a la par que con el monitoreo del margen de cargabilidad. Puede apreciarse que aun disponiendo de reservas de reactiva se puede alcanzar un colapso de tensión, lo que valida el hecho que para un nodo de carga existe un conjunto de generadores que afectan directamente el margen de estabilidad de tensión del mismo [26]. Esta evolución, graficada en despliegues basados en la teoría de la Conciencia Situacional (SA), permitiría que los operadores ejercieran acciones más acordes con las reservas de potencia reactiva, es decir, no se permitirían excesos de confianza ante la sensación de tenerse un buen respaldo de la tensión debido a que aún se tienen reservas de reactivos, cuando en realidad la situación está en camino al colapso. Obviamente, este alcance tiene que ver con el escenario de estabilidad de tensión de largo plazo [18].

Con el objetivo de reconocer la tendencia de cada indicador para los casos de estudio se presenta la Figura 9. En especial, puede notarse que la solución con el banco de capacitores está acorde con mejoras en las reservas de reactiva del sistema. También se presenta la Figura 10 para permitir observar el indicador del margen de cargabilidad para los casos. Es interesante la variación lineal del margen. Con una perspectiva adicional, a diferencia del indicador del margen de cargabilidad, el indicador de potencia reactiva de reserva expone una zona lineal seguida por otra no lineal, a medida que se incrementa la demanda de potencia. Esto sucede tanto para el caso base como para las contingencias. Este resultado podría ser aprovechado en caso de desear obtener el indicador por medio de métodos de regresión lineal, ahorrándose el cálculo previo de curvas $\mathrm{P}-\mathrm{V}$ y adaptándose a casos extraños no contemplados en los estudios de planeamiento. Como material de interés, la referencia [8] presenta un método de regresión lineal con otros indicadores y alcances.

Como complemento, la Figura 11 muestra la evolución de la tensión en la Barra 6 para los diferentes valores de potencia reactiva de reserva. 


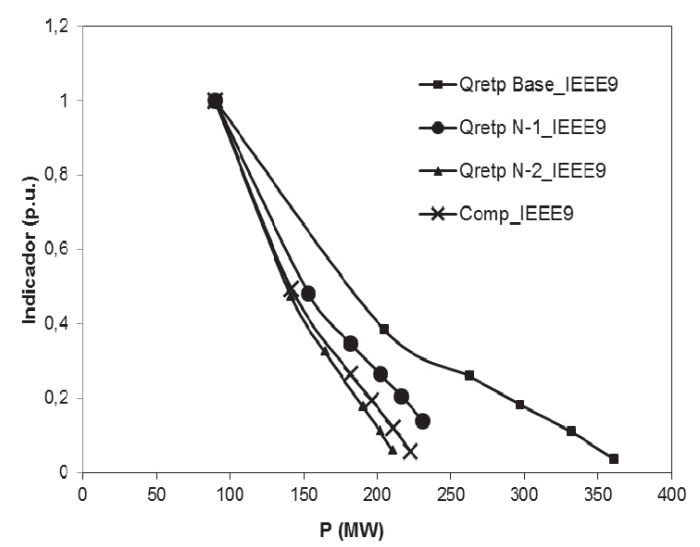

Figura 9. Indicador de reserva de potencia reactiva propuesto para los casos de estudio. Sistema IEEE 9 barras. Estrés en Barra 6.

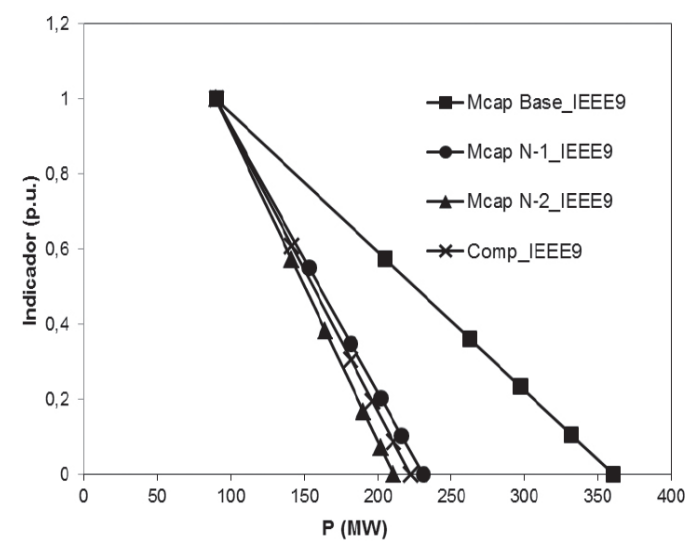

Figura 10. Indicador de margen de cargabilidad propuesto para los casos de estudio. Sistema IEEE 9 barras. Estrés en Barra 6.



Figura 11. Monitoreo de la tensión en Sistema IEEE 9 barras. Estrés en Barra 6.
Puede verificarse la relación de declive de las reservas totales y la tensión a medida que el sistema es estresado con el incremento de la demanda en la Barra 6.

La Figura 12 muestra la variación de los indicadores propuestos entre sí. Se aprecia una zona lineal al inicio y otra de pendiente diferente cerca del colapso. Esto es impulsado por la respuesta no lineal de las reservas de potencia reactiva para altas exigencias de carga.

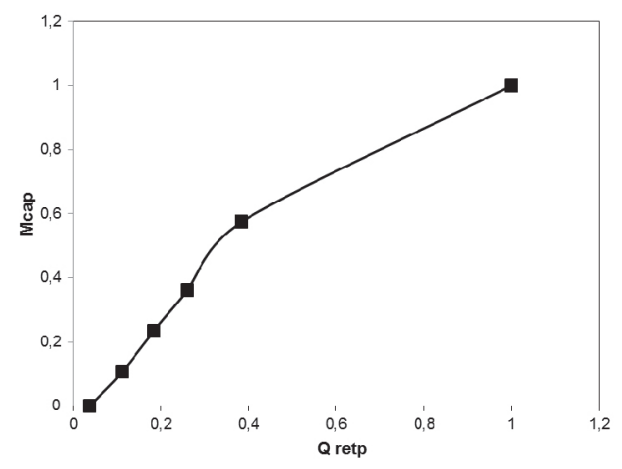

Figura 12. Monitoreo de la variación de los indicadores propuestos entre sí. Sistema IEEE 9 barras. Estrés en Barra 6.

Finalmente, la Figura 13 expone la variación del margen de cargabilidad (VSM del inglés Voltage Stability Margin) en función de las reservas de potencia reactiva. Como lo afirma [16], la relación entre las reservas de potencia reactiva posee en

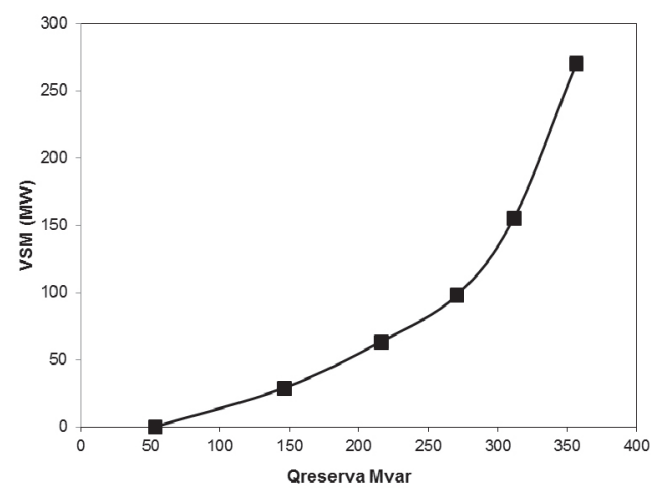

Figura 13. Monitoreo de la variación del margen de cargabilidad (VSM) en función de las reservas de potencia reactiva. Sistema IEEE 9 barras. Estrés en Barra 6. 
muchas ocasiones una característica cercanamente lineal, para al menos un rango de operación. La relación podría ser cuadrática a medida que el sistema se aproxima al punto de colapso.

\section{Sistema de Colombia [27]}

El sistema colombiano simulado tiene niveles de tensión de $500 \mathrm{kV}, 220 \mathrm{kV}, 115 \mathrm{kV}$ y otros menores para generación y distribución [27]. Posee alrededor de 2200 barras, 350 unidades de generación y 1500 ramas (líneas y transformadores). El escenario de operación es de máxima demanda. Las cargas totales poseen un valor alrededor de 11000 MW y 4000 Mvar. Según las características de los generadores, el margen de reserva de potencia reactiva total aproximado de los generadores para el caso base es de 6500 Mvar.

Se eligió una barra en una zona de mayor predominio de carga, hacia el sur-occidente del país. En dicha zona se estudió la Barra Mirolind en el nivel de 115 $\mathrm{kV}$. La carga base en dicha subestación es de 31 MW y 14,5 Mvar. En la Figura 14 se puede apreciar el sistema eléctrico aledaño a la Barra de interés.

Los análisis y comentarios a continuación serán breves, ya que se verá que las tendencias son básicamente las mismas del estudio de la red IEEE de 9 barras. Sin embargo, es una tarea de los operadores identificar barras críticas de acuerdo con su experiencia operativa, para ser estudiadas con los indicadores propuestos en el contexto de la estabilidad de tensión.

En la Figura 15 se pueden apreciar las curvas P-V para cuatro situaciones: el caso base, la contingencia de salida de una de las líneas entre las barras Mesa y Mirolind (N-1), la contingencia de salida de la otra línea paralela entre las mismas subestaciones ( $\mathrm{N}-2)$, $\mathrm{y}$ finalmente para el sistema de doble contingencia (N-2) con una compensación capacitiva de 50 Mvar que se conecta en la Barra Mirolind. Las curvas son coherentes con lo esperado para este tipo de análisis.

La Figura 16 presenta una evolución coherente con lo esperado para las reservas de potencia reactiva durante los estados de carga en cada caso de estudio.

Las Figuras 17 a 23 muestran la misma característica y tendencias que en el caso de la red IEEE de 9 barras, lo que confirma lo expuesto sobre la relación entre

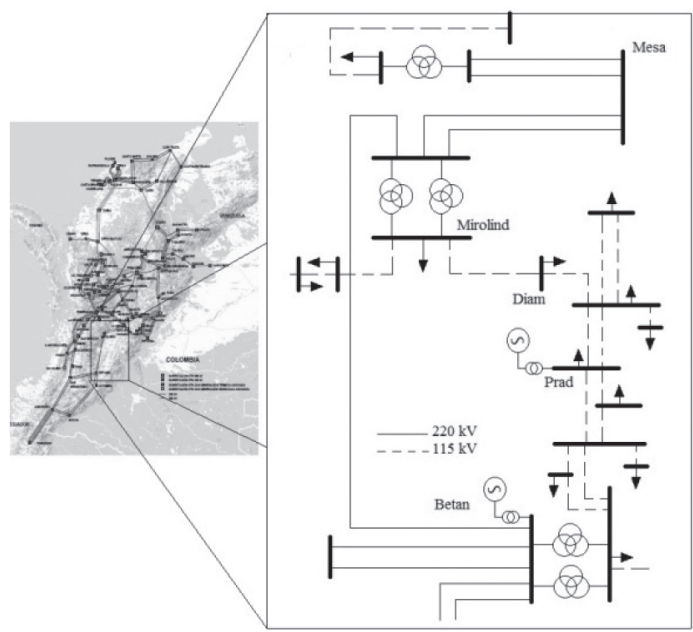

Figura 14. Sistema eléctrico de potencia colombiano. Ampliación zona de variación de la potencia [27].

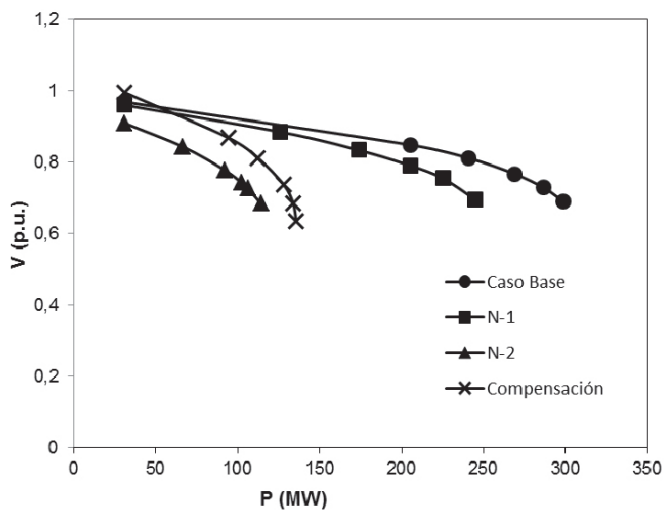

Figura 15. Curvas P-V sistema colombiano para los casos de estudio. Barra Mirolind.

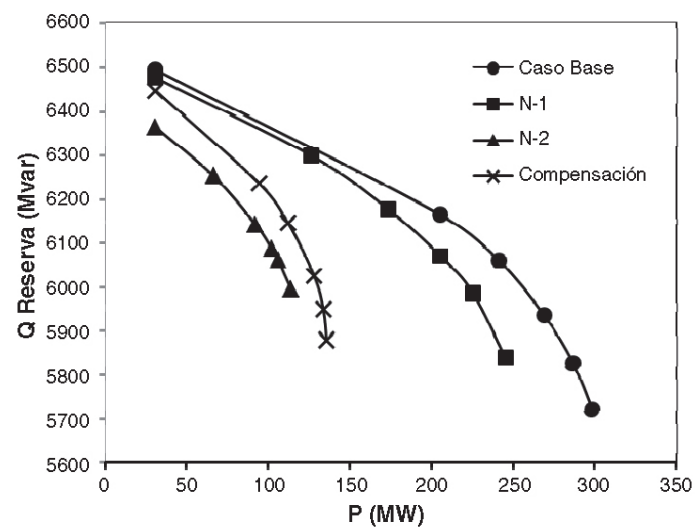

Figura 16. Potencias reactivas de reserva sistema colombiano para los casos de estudio. Estrés en Barra Mirolind. 


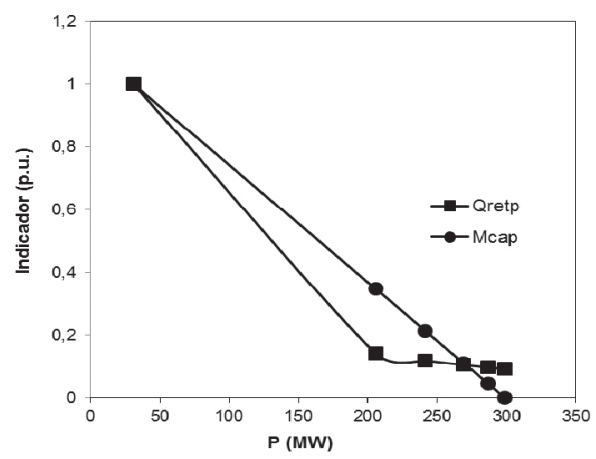

Figura 17. Comparación de indicadores para el caso base. Sistema colombiano. Estrés en Barra Mirolind.

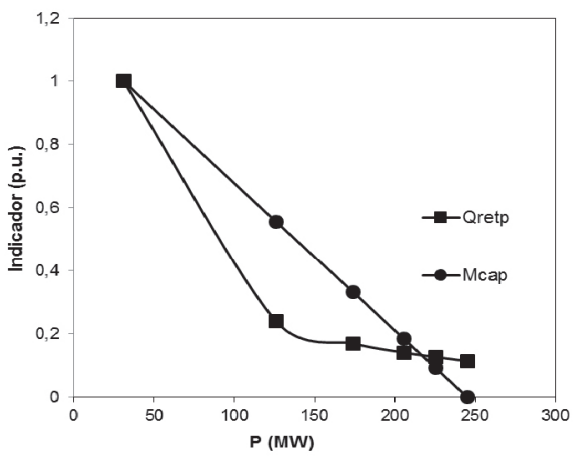

Figura 18. Comparación de indicadores para una contingencia. Sistema colombiano. Estrés en Barra Mirolind.

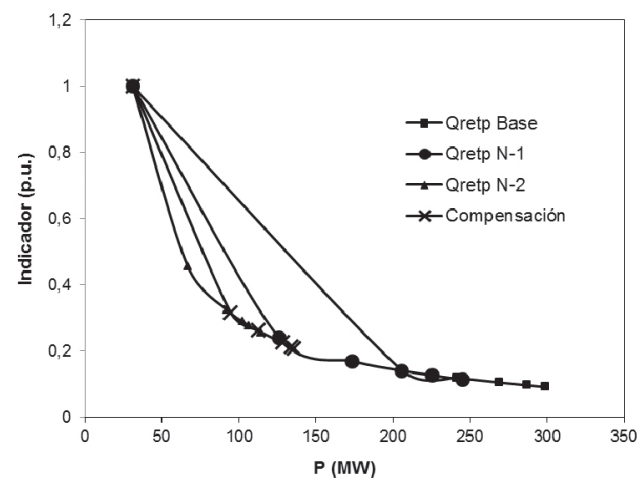

Figura 19 Indicador reserva potencia reactiva propuesto para casos de estudio. Sistema colombiano. Estrés en Barra Mirolind.

los indicadores de reservas de potencia reactiva y cargabilidad, además de la conveniencia para análisis en grandes redes eléctricas cuando se concentran los estudios en una barra específica.
En el caso de la Figura 19 puede notarse un comportamiento cuadrático más pronunciado que el caso de IEEE 9 barras. Esto va acorde con lo expuesto en [16] y en los análisis de la red IEEE de 9 barras.

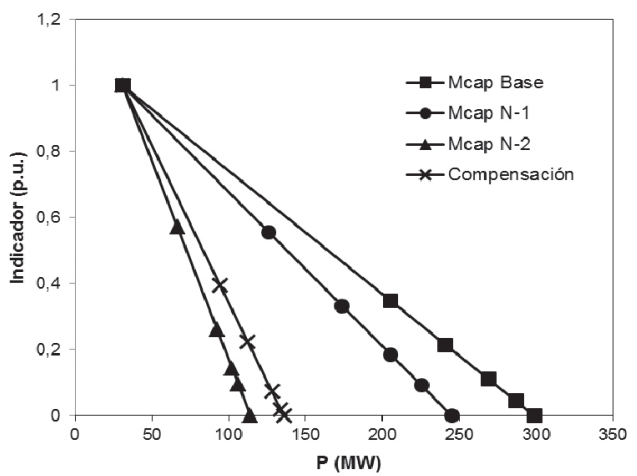

Figura 20. Indicador margen de cargabilidad propuesto para los casos de estudio. Sistema colombiano. Estrés en Barra Mirolind.

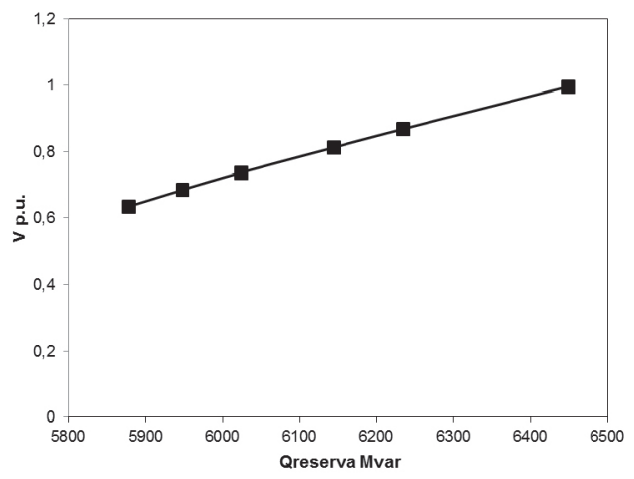

Figura 21. Monitoreo de tensión en Sistema colombiano. Estrés en Barra Mirolind.

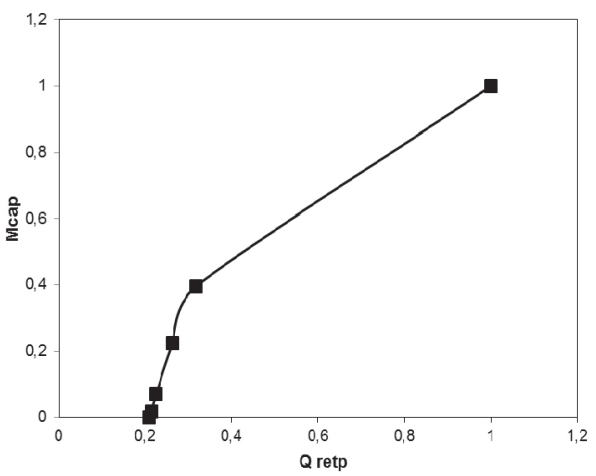

Figura 22. Monitoreo de la variación de los indicadores propuestos entre sí. Sistema colombiano. Estrés en Barra Mirolind. 


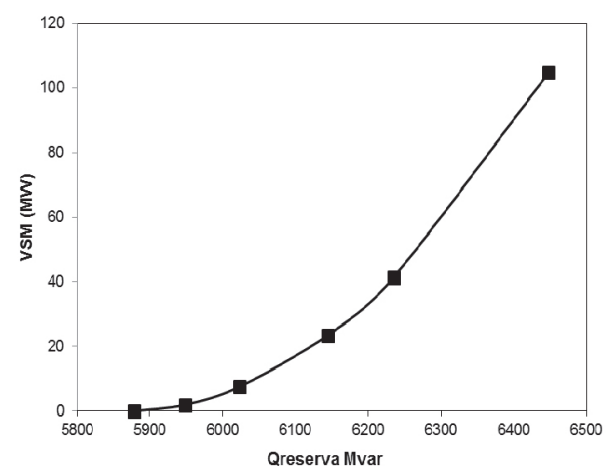

Figura 23. Monitoreo de la variación del margen de cargabilidad (VSM) en función de las reservas de potencia reactiva. Sistema colombiano. Estrés en Barra Mirolind.

\section{PROPUESTAS CON PMUS Y CONCIENCIA SITUACIONAL}

En esta sección se realiza una introducción a cómo se podrían programar los indicadores en un sistema que posee sincrofasores (PMU) y empleando la Conciencia Situacional.

El concepto de Conciencia Situacional (SA del inglés Situational Awareness), aplicado en la supervisión y visualización de sistemas de potencia, permite una constante vigilancia y monitoreo de las condiciones de un sistema.

La SA [10] posee tres etapas básicas: percepción de elementos en el entorno, comprensión de la situación y proyección de estados futuros.

Es la intención de la SA el observar un sistema complejo desde diversos entornos y de una manera global. Debido a lo complejo que son los sistemas de potencia y a su velocidad de cambio, los operadores deben estar en capacidad de predecir y manejar los cambios en los flujos de potencia; con una visión amplia de la estabilidad y con suficiente antelación. De esta forma, se reduce la probabilidad de caer en eventos en cascada o colapsos, cuando en realidad ya habría poco qué hacer para normalizar la operación.

Las herramientas o aplicaciones de un sistema de SA incluyen [28] tableros de control, monitoreo en tiempo real, control de estabilidad de frecuencia y programación de la separación de áreas si suceden ciertos eventos.
Los aplicativos y modelos de SA deben permitir identificar rápidamente las situaciones de riesgo y entregar al operador suficiente información que le ayude a interpretar la situación. Así, el operador o analista podría proyectar escenarios de posible ocurrencia ante riesgos identificados y sugerir correctivos para mitigar o solucionar los problemas.

Para cumplir con las tareas en el contexto de SA se debe integrar información histórica y de tiempo real, para conformar procesos predictivos.

En este orden de ideas, una herramienta de SA para el análisis de la estabilidad de tensión debe proveerse de diversas alternativas, basadas en indicadores complementarios entre sí y en visualizaciones de la evolución de la tensión para los estados de carga. Esta premisa se satisface con los indicadores propuestos y los gráficos tradicionales, algunos de ellos presentados en este artículo (curvas P-V, margen de cargabilidad, análisis modal, valores propios, reservas de potencia reactiva, etc.)

En la Figura 24 se esquematiza la forma en que podría implementarse un sistema de monitoreo de estabilidad de tensión en línea. Las PMU, ya sincronizadas entre sí mediante un sistema de posicionamiento global, toman las señales de las subestaciones, las cuales son básicamente valores de tensión, ángulo y frecuencia. Las medidas de los sincrofasores son enviadas por diversos medios, para ser recibidas,

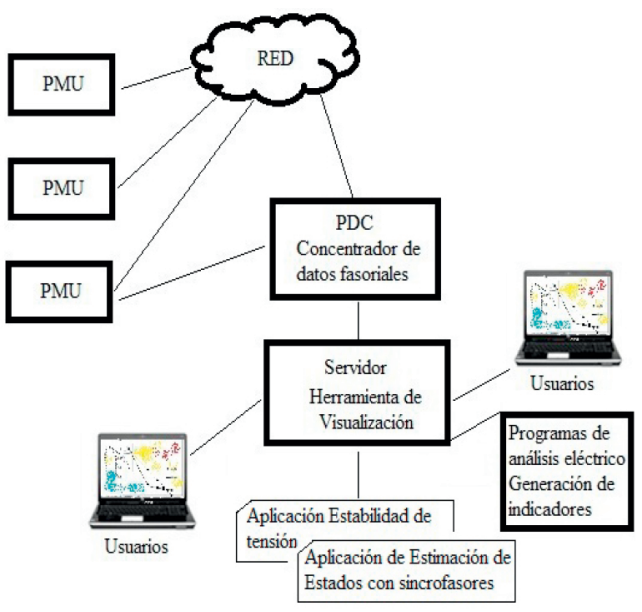

Figura 24. Propuesta de integración de indicadores de estabilidad de tensión con PMU y Conciencia Situacional. 
adecuadas y organizadas de forma coherente por el Concentrador de Datos Fasoriales (PDC del inglés Phasor Data Concentrator). Estas señales quedan ahora a disposición para ser utilizadas en diversas aplicaciones. En los centros de control local o distribuido, se debe disponer de un servidor que puede alojar las herramientas de visualización bajo el concepto de la SA. Los despliegues son llevados a mímicos o pantallas de los usuarios (analistas, operadores), una vez realizada una estimación de estados del sistema. Con la inclusión de los sincrofasores, la estimación de estados se puede realizar con mayor precisión y velocidad, siempre y cuando se disponga de una ubicación de PMU que permita la adecuada observabilidad del sistema.

El servidor interactúa con los programas de computador y aplicativos, entre ellos el de estabilidad de tensión. Los programas de análisis eléctrico permitirán generar los indicadores de estabilidad de tensión para cada estado de carga solicitado por los analistas y operadores. Ante un cambio topológico, un cambio en las condiciones de despacho o una contingencia se deberían recalcular los indicadores para monitorear la situación actual. Si una situación se identifica como adversa, debe evaluarse su solución mediante protocolos definidos previamente por el personal a cargo de la operación de la red. Por ejemplo, analizar las curvas de indicadores ante la conexión de bancos de capacitores, desconexión de reactores, cambiador de tomas bajo carga, limitación de cargas, etc.

Es importante informar (a continuación) el tiempo de ejecución básico para la construcción de indicadores para la red colombiana. El tiempo empleado fue de $30 \mathrm{~s}$ para calcular los indicadores utilizando el programa DIgSILENT PF v. 14.1 (x64). Se utilizó un computador con procesador intel i7, $2.9 \mathrm{GHz}$ de 8 GB de memoria RAM, con un sistema operativo Windows 7.

\section{CONCLUSIONES}

Se propusieron indicadores normalizados para análisis de estabilidad de tensión. Los indicadores permiten un análisis alternativo conjunto de la evolución de la tensión en barras de interés, en función de la potencia reactiva de reserva y del margen de cargabilidad.
Se encontró que los indicadores propuestos, concebidos en forma normalizada o en por unidad, son particularmente útiles o beneficiosos para analistas y operadores, ya que ayudan a que se interprete de manera adecuada si la presencia de potencia reactiva de reserva es o no, una garantía de la estabilidad de tensión para ciertos puntos del sistema.

Fue posible validar de una manera directa los comportamientos lineales en ciertos tramos de las variables relacionadas (reservas de energía reactiva y márgenes de potencia). Este comportamiento fue visto por otros autores mediante exhaustivos cálculos empleando técnicas de correlación. Para fines de operación, los comportamientos de los indicadores presentados poseen especiales potenciales de explotación dada la fácil percepción.

Los indicadores se aplicaron con éxito, exponiendo tendencias similares para una red IEEE pequeña ampliamente conocida y para una red grande de elevada cantidad de barras y generadores. También se pudo verificar que el comportamiento de los indicadores fue similar en cada red para las diferentes contingencias y modificaciones como en el caso de la instalación de compensación de reactivos. Es igualmente una tarea de los operadores identificar barras críticas de acuerdo con su experiencia operativa, para ser estudiadas con los indicadores propuestos en el contexto de la estabilidad de tensión.

De igual manera, mediante una arquitectura básica propuesta para la integración de tecnologías de información, se indicó la posibilidad del uso de indicadores para aplicaciones modernas de sincrofasores y Conciencia Situacional, en la que los operadores y analistas podrán contar con información precisa de lo que ocurre en el sistema y concebir probables soluciones con éxito.

\section{AGRADECIMIENTOS}

A la empresa XM E.S.P., operadora del mercado de energía de Colombia, por facilitar datos para análisis y consejos. Específicamente a W. Amador, R. León, G. Salazar, H. Sánchez, J. Gómez, J. Castaño y N. Duque. 


\section{REFERENCIAS}

[1] U.S.-Canada Power System Outage Task Force. "Final Report on the August 14th Blackout: Causes and Recommendations", pp. 5-220. April, 2004. Date of visit: February, 2012. URL: http://energy.gov/sites/prod/files/ oeprod/DocumentsandMedia/BlackoutFinalWeb.pdf

[2] C.W. Taylor. "Power System Voltage Stability". EPRI Power System Engineering Series, McGraw-Hill. 1st Ed. New York, USA, p. 273. 1994. ISBN: 0-07-063164-0.

[3] P. Kundur. "Power System Stability and Control". EPRI Power System Engineering Series, McGraw-Hill. 1st Ed. New York, USA, p. 1176. 1994. ISBN: 0-07-035958-X.

[4] V. Ajjarapu and C. Christy. "The continuation power flow: A tool for steady state voltage stability analysis". IEEE Transactions on Power Systems. Vol. 7, Issue 1, pp. 416-423. February, 1992. ISSN: 0885-8950. DOI: 10.1109/59.141737.

[5] T. Van Cutsem and C. Vournas. "Voltage Stability of Electric Power Systems". Norwell, MA, USA. Kluwer. 1st Ed. New York, USA, p. 378. 1998. ISBN: 978-0792381396.

[6] F. Dong, B. Chowdhury, M. Crow and L. Acar. "Improving voltage stability by reactive power reserve management". IEEE Transactions on Power Systems. Vol. 20, Issue 1, pp. 338-345. February, 2005. ISSN: 0885-8950. DOI: 10.1109/TPWRS. 2004.841241.

[7] B. Leonardi and V. Ajjarapu. "Development of multi-linear regression models for online voltage stability margin estimation". IEEE Transactions on Power Systems. Vol. 26, Issue 1, pp. 374-383. February, 2011. ISSN: 08858950. DOI: 10.1109/TPWRS.2010.2050155.

[8] B. Leonardi and V. Ajjarapu. "An Approach for Real Time Voltage Stability Margin Control via Reactive Power Reserve Sensitivities". IEEE Transactions on Power Systems. Vol. 28, Issue 2, pp. 615-625. April, 2013. ISSN: 08858950. DOI: 10.1109/TPWRS.2012.2212253.

[9] C. Affonso, L. da Silva, F. Lima and S. Soares. "MW and MVar Management on Supply and Demand Side for Meeting Voltage Stability Margin Criteria". IEEE Transactions on Power Systems. Vol. 19, Issue 3, pp. 1538-
1545. August, 2004. ISSN: 0885-8950. DOI: 10.1109/TPWRS.2004.831659.

[10] J. Giri, M. Parashar, T. Josh and M. Vahid. "The Situation Room: Control Center Analytics for Enhanced Situational Awareness". IEEE Power and Energy Magazine. Vol. 10, Issue 5, pp. 24-39. September, 2012. ISSN: 15407977. DOI: 10.1109/MPE.2012.2205316.

[11] R. Bobba, J. Dagle, E. Heine, H. Khurana, W. Sanders, P. Sauer and T. Yardley. "Enhancing Grid Measurements: Wide Area Measurement Systems, NASPInet, and Security". IEEE Power and Energy Magazine. Vol. 10, Issue 1, pp. 67-73. January, 2012. ISSN: 1540-7977. DOI: 10.1109/MPE.2011.943133.

[12] R. Diao, V. Vittal and N. Logic. "Design of a Real-Time Security Assessment Tool for Situational Awareness Enhancement in Modern Power Systems". IEEE Transactions on Power Systems. Vol. 25, Issue 2, pp. 957965. May, 2010. ISSN: 0885-8950. DOI: 10.1109/TPWRS.2009.2035507.

[13] D. Trudnowski, J. Pierre, N. Zhou, J. Hauer and M. Parashar. "Performance of Three Mode-Meter Block-Processing Algorithms for Automated Dynamic Stability Assessment". IEEE Transactions on Power Systems. Vol. 23, Issue 2, pp. 680-690. May, 2008. ISSN: 08858950. DOI: 10.1109/TPWRS.2008.919415.

[14] V. Kekatos, G. Giannakis and B. Wollenberg. "Optimal Placement of Phasor Measurement Units via Convex Relaxation". IEEE Transactions on Power Systems. Vol. 27, Issue 3, pp. 1521-1530. August, 2012. ISSN: 08858950. DOI: 10.1109/TPWRS.2012.2185959.

[15] J. Zhu, E. Zhuang, C. Ivanov and Z. Yao. "A Data-Driven Approach to Interactive Visualization of Power Systems". IEEE Transactions on Power Systems. Vol. 26, Issue 4, pp. 2539-2546. November, 2011. ISSN: 0885-8950. DOI: $10.1109 /$ TPWRS.2011.2119499.

[16] L. Bao, Z. Huang and W. Xu. "Online voltage stability monitoring using VAR reserves". IEEE Transactions on Power Systems. Vol. 18, Issue 4, pp. 1461-1469. November, 2003. ISSN: 0885-8950. DOI: 10.1109/ TPWRS.2003.818706.

[17] IEEE Task Force on Excitation Limiters. "Recommended models for overexcitation limiting devices". IEEE Transactions on 
Energy Conversion. Vol. 10, Issue 4, pp. 706713. December, 1995. ISSN: 0885-8969. DOI: $10.1109 / 60.475843$.

[18] P. Kundur, J. Paserba, V. Ajjarapu, G. Andersson, A. Bose, C. Canizares, N. Hatziargyriou, D. Hill, A. Stankovic, C. Taylor, T. Van Cutsem and V. Vittal. "Definition and classification of power system stability". IEEE Transactions on Power Systems. Vol. 19, Issue 4, pp. 13871401. November, 2004. ISSN: 0885-8950. DOI: $10.1109 /$ TPWRS.2004.825981.

[19] B. Gao, K. Morison and P. Kundur. "Voltage stability evaluation using modal analysis". IEEE Transactions on Power Systems. Vol. 7, Issue 4, pp. 1529-1542. November, 1992. ISSN: 0885-8950. DOI: 10.1109/59.207377.

[20] L. Franchi, M. Innorta, P. Marannino and C. Sabelli. "Evaluation of economy and or security oriented objective functions for reactive scheduling in large scale systems". IEEE Transactions on Power Apparatus and Systems. Vol. PAS-102, Issue 10, pp. 34813488. October, 1983. ISSN: 0885-8950. DOI: 10.1109/TPAS.1983.317847.

[21] IEEE Std C37.118.1-2011. "IEEE Standard for Synchrophasor Measurements for Power Systems". IEEE. New York, USA, p. 49. 2011. ISBN: 978-0-7381-6811-1.

[22] IEEE Std C37.118.2-2011. "IEEE Standard for Synchrophasor Data Transfer for Power Systems". IEEE. New York, USA, p. 43. 2011. ISBN: 978-0-7381-6813-5.
[23] G. Valverde, S. Chakrabarti, E. Kyriakides and V. Terzija. "A Constrained Formulation for Hybrid State Estimation". IEEE Transactions on Power Systems. Vol. 26, Issue 3, pp. 11021109. August, 2011. ISSN: 0885-8950. DOI: 10.1109/TPWRS. 2010.2079960.

[24] P.M. Anderson and A.A. Fouad. "Power System Control and Stability". IEEE Press. Second edition. New Jersey, USA, p. 672. 2002. ISBN: 978-0-471-23862-1.

[25] DIgSILENT GmbH. "DigSILENT Power Factory Version 14.1 Manual”. DIgSILENT GmbH. Gomaringen, Germany. Vol. 2, p. 550. 2011.

[26] R. Schlueter. "A voltage stability security assessment method". IEEE Transactions on Power Systems. Vol. 13, Issue 4, pp. 14231438. November, 1998. ISSN: 0885-8950. DOI: $10.1109 / 59.736286$.

[27] Ministerio de Minas y Energía, Unidad de Planeación Minero Energética, UPME. "Plan de Expansión de Referencia Generación - Transmisión 2012-2025”. República de Colombia - UPME, p. 187. Bogotá, Colombia. 2012.

[28] T. Gentile, J. Chow, V. Balasubramanian, D. Sobajic, L.M. Beard and D. Dinh. "NASPI process applied to locate Phasor Measurement Units (PMUs) within the New York Control Area (NYCA)". IEEE Power and Energy Society General Meeting, San Diego 2012. San Diego, CA, USA. July, 2012. 